\section{DNA DEMETHYLATION}

\section{IncRNA express delivery}

Mol. Cell 55, 604-614 (2014)

DNA methylation is a reversible epigenetic process that is associated with transcriptional repression. Methyl groups are installed at the C5 positions of cytosines in CpG-rich sequences by DNA methyltransferase enzymes, but the machineries that remove these marks remain incompletely characterized. Arab et al. now provide evidence that gene-specific DNA demethylation may be directed by long noncoding RNAs (lncRNAs) and adaptor proteins that recruit the DNA demethylation apparatus. Following up on their earlier studies of the tumor suppressor, transcription factor 21 (TCF21), the authors discovered a promoter at a downstream $\mathrm{CpG}$ island within the TCF21 gene that expresses a 4.5-kb RNA nuclear antisense transcript that has features of a lncRNA. Expression of this transcript, termed TCF21 antisense RNA inducing demethylation (TARID), leads to decreased DNA methylation at the transcriptional start site (TSS) of TCF21 and increased expression of TCF21 mRNA. Immunoprecipitation studies revealed that TARID binds GADD45A, an RNA-binding protein known to regulate DNA demethylation, and also the TCF21 promoter through a complementary antisense interaction with TSS sequences. Further biochemical experiments showed that this ribonucleoprotein complex recruits two enzymes linked to active DNA demethylation-thymine DNA glycosylase and ten-eleven translocation methylcytosine dioxygenase-to the TCF21 promoter. Taken together, these data suggest that TARID, and perhaps other lncRNAs, may serve as sequence-specific bar codes to address the DNA demethylation machinery to specific gene loci.

\section{NANOWIRES}

\section{String me along}

Proc. Natl. Acad. Sci. USA

doi:10.1073/pnas.1410551111

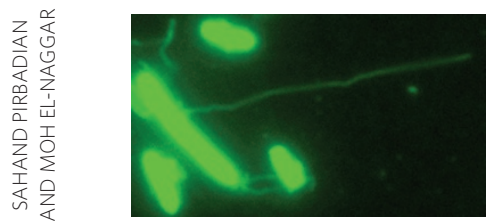

Metal-reducing bacteria use extracellular nanowires to send electrons to external metal acceptors. However, the mechanisms involved in generating these wires are not clear, in part because characterization of these wires has been conducted under nonphysiological conditions and in part because the molecular composition of the wires remains unknown. Pirbadian et al. now report measurements using live cells that shed light on these intriguing structures. Fluorescence imaging of Shewanella oneidensis MR-1 upon $\mathrm{O}_{2}$ limitation using a protein-dependent dye highlighted filament formation and production of membrane vesicles, in agreement with previous work, whereas labeling with a redox-sensing dye showed that filament formation occurred with an increase in cellular reductase activity. Though previous proposals had suggested that nanowires are constructed using pilus proteins, expression of pilin genes did not increase during filament formation. Instead, the use of a lipid bilayer dye and fluorescent protein fusions demonstrated that the filaments include both

\section{ER STRESS}

\section{Redox trade-off}

membranes and periplasmic components, suggesting that the wires are outer membrane extensions. The authors confirmed that outer membrane cytochromes-previously shown to be required for electron transport by $S$. oneidensis - were not only expressed in higher amounts upon $\mathrm{O}_{2}$ limitation but also located along the wires. AFM images collected at different time points captured a nanowire maturation process that progressed from vesicle chains to continuous filaments. Given the common structural elements used to form the nanowires, the authors hypothesize that additional extracellular extensions might await discovery.

LIPIDS

\section{Flexible fill-ins}

Science 345, 693-697 (2014)

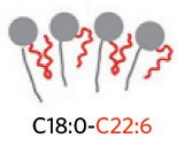

Phospholipids (PLs) containing polyunsaturated acyl chains have functional effects on specialized membranes such as synaptic vesicles due to their ability to make membranes more malleable. To gain new insights into the functions of polyunsaturated PLs in membrane fission, Pinot et al. examined the effect of varying the ratio between mono- and polyunsaturated PLs on the function of the fission machinery-consisting of endophilin and the GTPase dynaminon model membranes. Polyunsaturated PLs induced faster hydrolysis of GTP by dynamin and eliminated dynamin's sharp response to membrane curvature. Electron and light microscopy showed that polyunsaturated PLs sensitize membranes to the mechanical activities of the fission machinery. Using membrane deformation agents and a specific dynamin inhibitor, the authors found further evidence that polyunsaturated PLs facilitate endocytic events under the control of the fission machinery. They also found that polyunsaturated PLs more effectively decreased the bending rigidity of the plasma membrane of cultured cells with defined fatty acids in experiments using optical tweezers. Molecular dynamics simulations and experiments using lipid packing sensors suggested a mechanism by which polyunsaturated PLs decrease the bending rigidity and lower the force threshold for generation of fission tubules-by using their flexible chain to adapt their conformation on the basis of the curvature of the membrane. 